\title{
Intrabunch motion
}

\author{
E. Métral* \\ CERN, 1211 Geneva, Switzerland
}

(Received 24 July 2020; accepted 4 January 2021; published 22 January 2021)

\begin{abstract}
Impedance-driven (but not only) coherent beam instabilities are usually studied analytically with the linearized Vlasov equation, ending up with an eigenvalue system to solve. The eigenvalues describe the beam oscillation mode-frequency shifts, leading in particular to intensity thresholds defined by the longitudinal mode coupling instability in the longitudinal plane and by the transverse mode coupling instability in the transverse plane in the absence of chromaticity. This can be directly compared to measurements in particular for the lowest modes and in the absence of tune spread. In the presence of nonlinearities or when higher-order modes are involved, this becomes quite difficult, if not impossible, and the coupling between the modes cannot be directly measured (or simulated) anymore. Another important observable is the intrabunch motion, which can be also accessed analytically thanks to the eigenvectors. To the author's knowledge, until now, the intrabunch signal has only been explained theoretically for independent longitudinal or transverse beam oscillation modes, i.e., when the bunch intensity is sufficiently low compared to the mode coupling threshold. It was never explained theoretically in detail when two (or more) modes are involved. For instance, no answers were already given to these questions: is (are) there some fixed point(s) when the transverse mode coupling instability starts? If yes, where is it (are they)? And what happens in the presence of mode decoupling? Any number of modes can be treated with the general approach discussed in this paper, which is based on the GALACTIC Vlasov solver (which was previously successfully benchmarked against the PyHEADTAIL macroparticle tracking code as concerns the beam oscillation mode-frequency shifts). However, to be able to clearly see what happens when the bunch intensity is increased, the simple case of two modes is discussed in detail. The purpose of this paper is to describe the different regimes, below, at, above the transverse mode coupling instability and also after the mode decoupling (as it happens sometimes), using a simple analytical model (where two modes are considered together), which helps to really understand what happens at each step. Better characterizing an instability is the first step before trying to find appropriate mitigation measures and push the performance of a particle accelerator. The evolution of the intrabunch motion with intensity is a fundamental observable with high-intensity high-brightness beams.
\end{abstract}

DOI: 10.1103/PhysRevAccelBeams.24.014401

\section{INTRODUCTION}

The intrabunch signal for independent longitudinal or transverse beam oscillation modes, i.e., at sufficiently low intensity, has been explained analytically for impedancedriven coherent beam instabilities already several decades ago by Laclare in [1], and it has been observed and confirmed in many machines and macroparticle tracking simulation codes. In this case, applying the Vlasov equation to first order, the motion of the beam is described by a superposition of modes rather than a collection of

\section{*Elias.Metral@cern.ch}

Published by the American Physical Society under the terms of the Creative Commons Attribution 4.0 International license. Further distribution of this work must maintain attribution to the author(s) and the published article's title, journal citation, and DOI. individual particles, and one ends up with an eigenvalue system to solve (the interested reader could have all the details for instance in [2] and references therein). The result is an infinite number of modes of oscillation $m q$ (as there are 2 degrees of freedom, the longitudinal amplitude and phase), with $m$ the azimuthal mode number and $q$ the radial one. The latter is defined as $q=|m|+2 k$ (with $k$ an integer between 0 and infinity): with this definition, the radial mode number $q$ represents the number of nodes of the superimposed (turn after turn) intrabunch signals, which is a usual observable in particle accelerators. The first radial mode corresponds to $k=0$ and therefore $q=|m|$ : in this case, and to simplify the notation, the mode $m|m|$ is usually written with only one number, the azimuthal mode number $m$. Examples of such intrabunch signals (superimposed turn after turn) are shown in Fig. 1 (left) for the case of the first two modes, 0 and -1 . It can be seen, in particular, that these signals exhibit a clear left/right (head/tail) symmetry and 

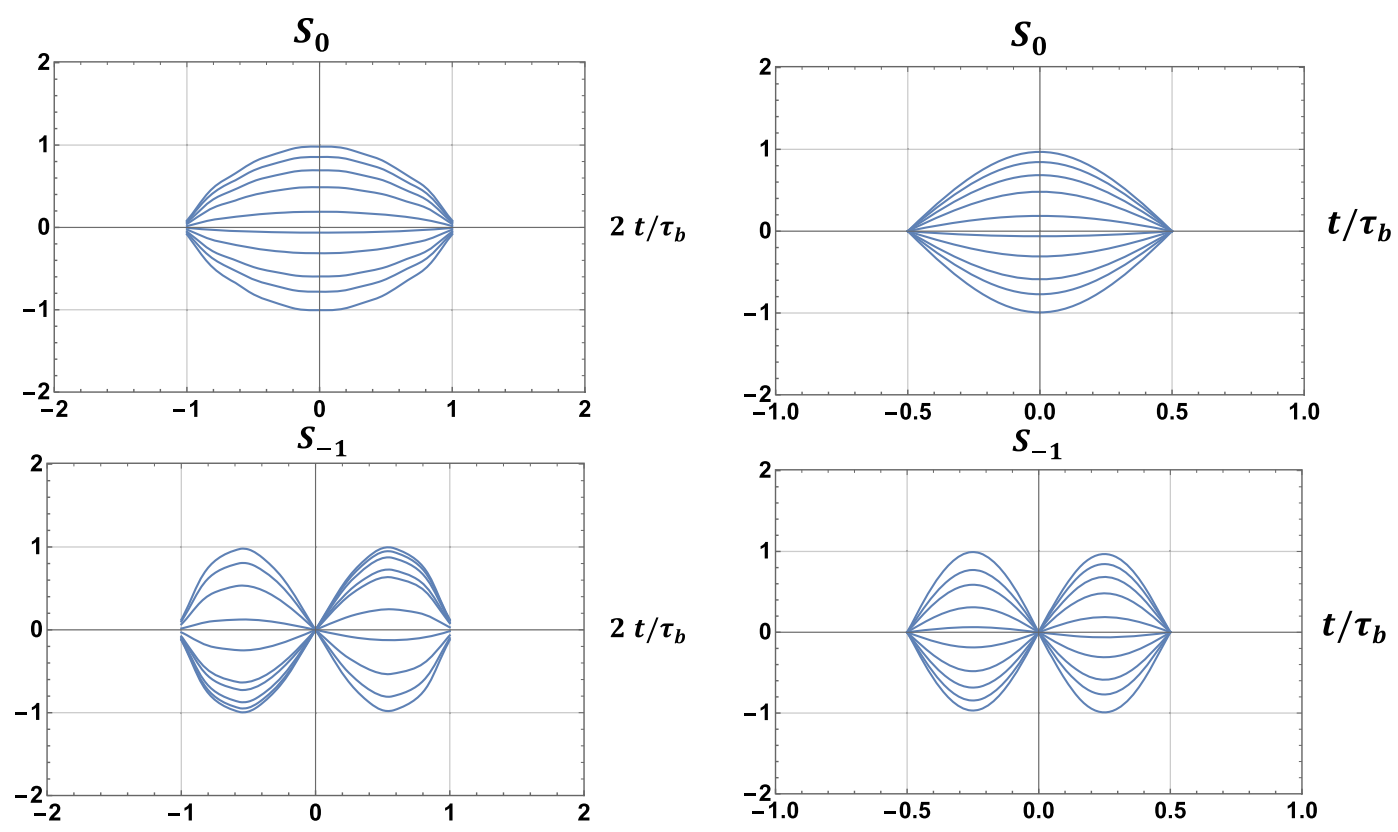

FIG. 1. Left: intrabunch signal deduced from the solutions of the eigenvalue problem at low intensity using the GALACTIC Vlasov solver for the case of a bunch with a longitudinal "water-bag" distribution [1] interacting with a purely inductive impedance [2]; right: approximation by sinusoidal modes given by Eqs. (1) and (2) [3]. Here, $t$ is the time, $\tau_{b}$ is the full (4-sigma) bunch length and the head of the bunch is on the left while the tail is on the right.

they are called standing-wave patterns. As can be seen in Fig. 1 (right) and as discussed by Sacherer in [3], these intrabunch signals can be well approximated by

$$
\begin{aligned}
S_{0}(t, n) & =\cos \left(\frac{\pi t}{\tau_{b}}\right) \cos (2 \pi n Q), \\
S_{-1}(t, n) & =\sin \left(\frac{2 \pi t}{\tau_{b}}\right) \cos (2 \pi n Q),
\end{aligned}
$$

where $t$ is the time, $n$ the turn number, $\tau_{b}$ the full (4-sigma) bunch length and $Q$ the transverse tune.

However, many pictures measured in particle accelerators or simulated with macroparticle tracking codes are not left/right symmetric, such as e.g., Fig. 2, which has been obtained through single-bunch PyHEADTAIL [4] macroparticle tracking simulations with the CERN super proton synchrotron (SPS) transverse impedance model [5]. Therefore, how can we understand theoretically such a picture and in particular the left/right asymmetry?

To try and understand this observed asymmetry, I proposed few years ago to Amorim to study numerically, with the DELPHI Vlasov solver [6], the intrabunch motion in the presence of two (or more) modes, which revealed different regimes, well below the transverse mode coupling instability (TMCI) intensity threshold [7,8], close to the TMCI intensity threshold and above the TMCI intensity threshold [9].

The TMCI instability is supposed to be well known, as it was first described four decades ago, in 1980, by
Kohaupt [10] in terms of coupling of Sacherer's head-tail modes, extending to the transverse motion, the theory proposed by Sacherer to explain the longitudinal microwave instability. Furthermore, it has been the subject of extensive experimental observations. However, only the evolution of the modes with intensity was studied in detail in the past (to identify in particular the intensity threshold when the modes couple) but the evolution of the intrabunch motion with intensity was never analyzed in detail in the presence of two (or more) modes. This is striking for instance if one looks at the two famous textbooks from Chao [11] or $\mathrm{Ng}$ [12] (and references therein), where this important observable is not discussed. Nothing could be found also in the recent publications despite the fact that TMCI instabilities can be observed in the presence of beam coupling impedance but also in the presence of beambeam [13], electron cloud [14] and space charge [15]. The intrabunch motion was discussed a bit more recently, in particular by Burov, in the framework of the studies of the effect of space charge on TMCI [16], with the presence of convective instabilities under some assumptions [17]. However, none of the pictures produced in this paper and their explanations could be found there. Furthermore, only the case of an impedance-driven TMCI is treated here, even if it should certainly apply also to other kinds of TMCI instabilities, as what matters here is the coupling of transverse modes (whatever the origin of these modes).

The evolution of the intrabunch motion with intensity is another way of looking at the TMCI instability, which can be characterized by a traveling-wave pattern along the 


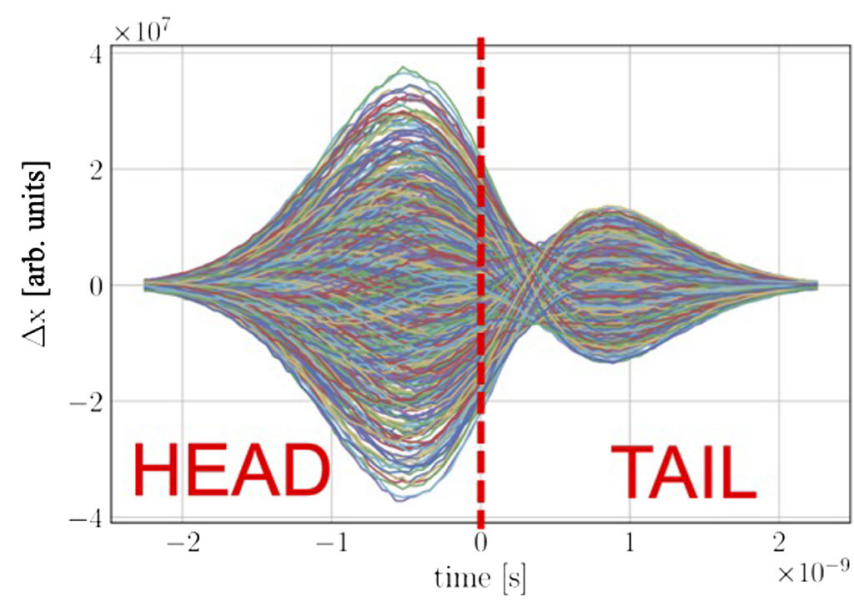

FIG. 2. Simulated (with the PyHEADTAIL macroparticle tracking code, using a Gaussian longitudinal profile) transverse singlebunch instability with the CERN SPS transverse impedance model and a chromaticity close to zero $\left(Q^{\prime}=3\right)$. The amplitude of the instability is plotted vs time with respect to the center of the bucket over 2000 consecutive turns. This figure is courtesy of Beck [5].

bunch, as a result of the coupling of two standing-wave patterns (head-tail modes). It is true that the general case considering the interaction between two (or several) modes is more involved than the low-intensity case as it now depends on the bunch intensity. For instance, in the example treated in [9] with only the two modes 0 and -1 , the intrabunch signal of Fig. 3 has been numerically obtained when the two modes 0 and -1 start to couple. It is worth noting that all the information should be already available in all the Vlasov solvers: it is just that this information was not exploited much until now as the intrabunch motion was mainly used to characterize headtail instabilities, where all the modes can be treated independently. Indeed, in this case the intrabunch motion

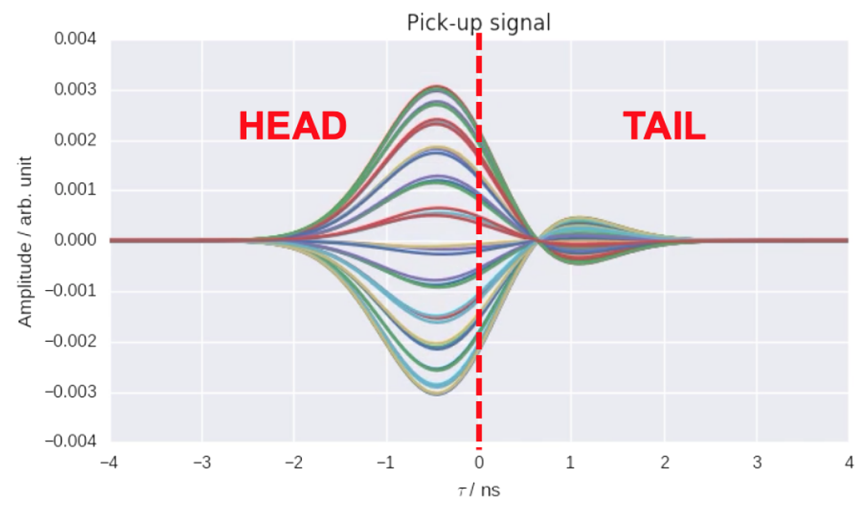

FIG. 3. Simulated (with the DELPHI Vlasov solver, using a Gaussian longitudinal profile) transverse intrabunch signal (superimposed turn after turn), as observed from a pickup monitor, for the case of a bunch interacting with an impedance and for the chromaticity $Q^{\prime}=0$, when the two modes 0 and -1 start to couple. This figure is courtesy of Amorim [9]. is usually the most used observable in accelerators and it helps to characterize the instability before trying to find the appropriate mitigation measures, as the later differ depending on the type of instability. For instance, in the case of a head-tail instability one will often try to introduce a certain amount of nonlinearities to provide some Landau damping whereas in the case of a TMCI instability one will not do this as the required tune spread is huge (of the order of the synchrotron tune, which might lead to other issues).

The motivation for this study was the following: how can we understand theoretically the observed asymmetries of Figs. 2 and 3 and for instance the fixed point of Fig. 3 when the two modes start to couple? The purpose of this paper is to answer these questions (and more), to explain theoretically and in detail the evolution of the intrabunch signal when both modes 0 and -1 are considered together, in an example case where both modes couple at some bunch intensity and then decouple at another one [18] (similarly to the case discussed in [9]). Of course, in the general case several modes might be needed to be able to quantitatively describe the real situation but then it would only be a matter of summing over more modes: the physics would have been already fully understood (as can be observed in [9]).

\section{GENERAL APPROACH WITH THE GALACTIC VLASOV SOLVER}

The Galactic Vlasov solver has been explained in detail in [2] for the case of a single bunch interacting with any impedance. In particular, the following two equations need to be solved

$$
\begin{gathered}
\sigma(l)=\sum_{i, j=-\infty}^{\infty} a_{i j} \sigma_{i j}(l), \\
\frac{\Delta Q}{Q_{s}} a_{k l}=H a_{i j},
\end{gathered}
$$

where the (general, high-intensity) eigenvector $\sigma$ is decomposed on the low-intensity eigenvectors $\sigma_{i j}$, with the coefficients $a_{i j}$, which can be identified by finding the eigenvectors of the eigenvalue system of Eq. (4), $l$ is an integer, $\Delta Q$ the (complex) betatron tune shift, $Q_{s}$ the synchrotron tune and $H$ is the matrix to be diagonalized (which includes in particular the bunch intensity and the impedance: the interested reader can find all the details in [2]) but most of the times, one only looks at the evolution of the modes and the instability growth rate with the bunch intensity (given by the real and imaginary parts of the tune shifts) [2] and not at the intrabunch signal when several modes are involved. To the author's knowledge, the intrabunch signal was never explained theoretically in detail when two (or more) modes are involved, and for instance the position of the fixed point of Fig. 3 was not explained yet. From Eqs. (3) and (4), it can be seen that once the 
coefficients $a_{i j}$ are obtained, the intrabunch signal can be plotted following [1], using the eigenvector $\sigma$ [as discussed before in the case of low-intensity independent head-tail modes, see Fig. 1 (left)]. Any number of modes can be treated with this approach, but to be able to clearly see what happens when the bunch intensity is increased, the simple case of two modes is discussed in the next section.

\section{SIMPLE MODEL CONSIDERING BOTH MODES 0 AND - 1 TOGETHER}

It is worth mentioning first that the GALACTIC Vlasov solver, used in this paper, was benchmarked against the PyHEADTAIL macroparticle tracking code, and an excellent agreement was reached, as can be seen from Figs. 15 and 16 of Ref. [2]. Furthermore, it is also worth observing from these figures that they correspond to the case of a first mode coupling instability between modes 0 and -1 and then a mode decoupling (before another mode coupling takes place between higher-order modes). This case is therefore very close to the situation, which is considered in this paper, and which is based on the simplified model used in the past to study the destabilizing effect of the LHC transverse damper (or any resistive bunch-by-bunch transverse damper) for zero chromaticity (deduced from studies with the GALACTIC Vlasov solver) but without damper $[19,20]$. One considers thus the case of a bunch (with a longitudinal "water-bag" distribution [1]) interacting with a broadband resonator impedance with a quality factor of 1 and a resonance frequency $f_{r}$ such that $f_{r} \tau_{b}=0.8$. This case was first treated exactly in [19], as can be observed from Fig. 2 of Ref. [19]. Then, trying to understand the detailed physical mechanism behind the destabilizing effect of a resistive transverse damper, it was observed that the physics could be fully understood by considering only the two modes 0 and -1 (over the relevant intensity range), as can be seen by comparing Figs. 2 and 3 of Ref. [19]. In this case the matrix, which needs to be diagonalized, can be approximated by [as can be seen from Eq. (7) of Ref. [19] ]

$$
H=\left[\begin{array}{cc}
-1 & -0.23 j x \\
-0.55 j x & -0.92 x
\end{array}\right]
$$

with $j$ the imaginary unit and $x$ a normalized parameter proportional to the bunch intensity [19]. Note that this matrix was obtained from numerical computations with the GALACTIC Vlasov solver, and that it can take several forms: this explains why the off-diagonal terms are complex here, instead of being real, but this leads to the same results as it is the product between the two which matters. The related eigenvalues are depicted in Fig. 4, while the eigenvectors are represented in Fig. 5. As can be seen from Fig. 4, the two modes 0 and -1 couple at $x=0.61$, which defines the TMCI intensity threshold. Below this value, the bunch is stable, while above it the bunch is unstable (as can be also observed from the red curve exhibiting a nonzero value).

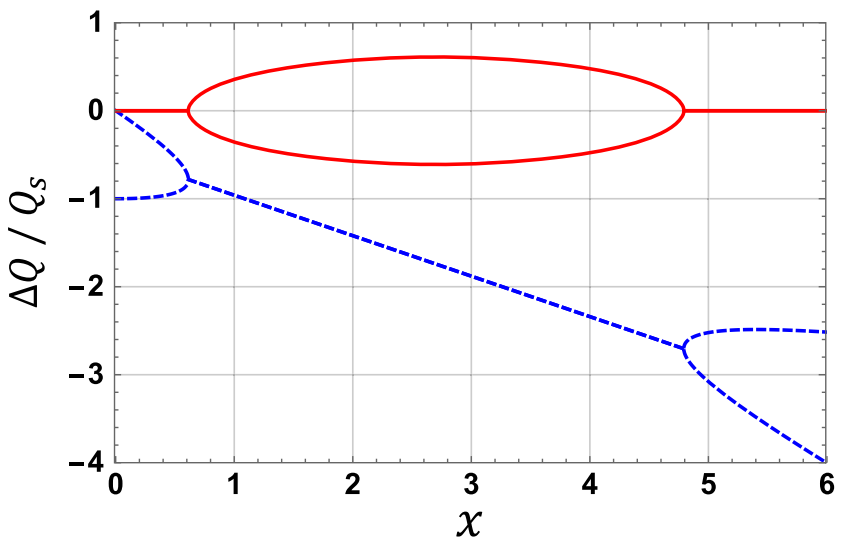

FIG. 4. Eigenvalues of the matrix of Eq. (5) with $x$ a normalized parameter proportional to the bunch intensity [19]: real part in blue (dashed line) and imaginary part in red (full line).

When $x=4.8$, the two modes decouple and the bunch is stable again.

Below the TMCI intensity threshold, and considering the approximated sinusoidal modes of Eqs. (1) and (2) as discussed before (see Fig. 1), the intrabunch signal $S(t, n)$ is given by (exchanging the coefficients for each mode)

$$
S(t, n) \propto a_{0} S_{0}(t, n)-a_{-1} S_{-1}(t, n),
$$

with $a_{0}$ and $a_{-1}$ given by the (dashed) blue curve in Fig. 5, which depend on the bunch intensity. At very low bunch intensity, the signals of Fig. 1 are recovered (see Fig. 6) and the two modes are independent and not perturbed by the other one. As the bunch intensity increases, the mode 0 is more and more perturbed by the mode -1 and vice versa (see Fig. 7).

At the TMCI intensity threshold, $S(t, n)$ is given by

$$
S(t, n) \propto a\left[S_{0}(t, n)-S_{-1}(t, n)\right]
$$

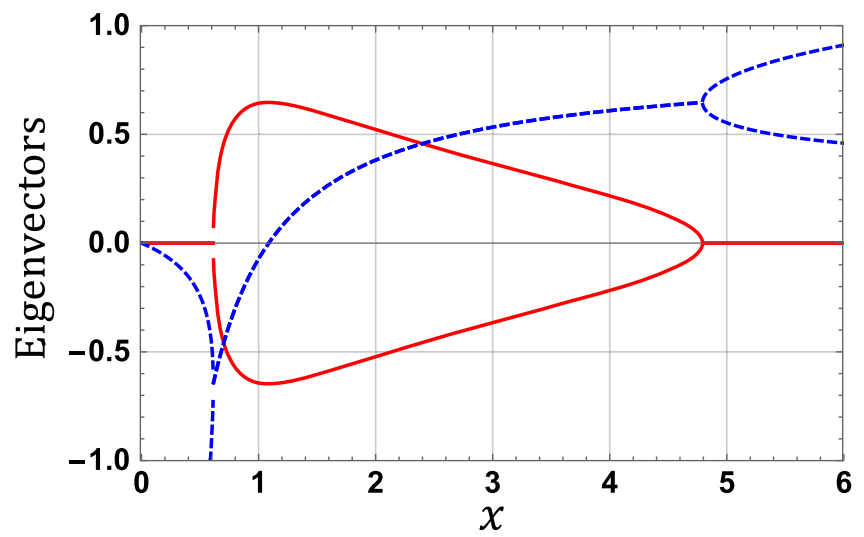

FIG. 5. Eigenvectors of the matrix of Eq. (5) with $x$ a normalized parameter proportional to the bunch intensity [19]: imaginary part in blue (dashed line) and real part in red (full line). 

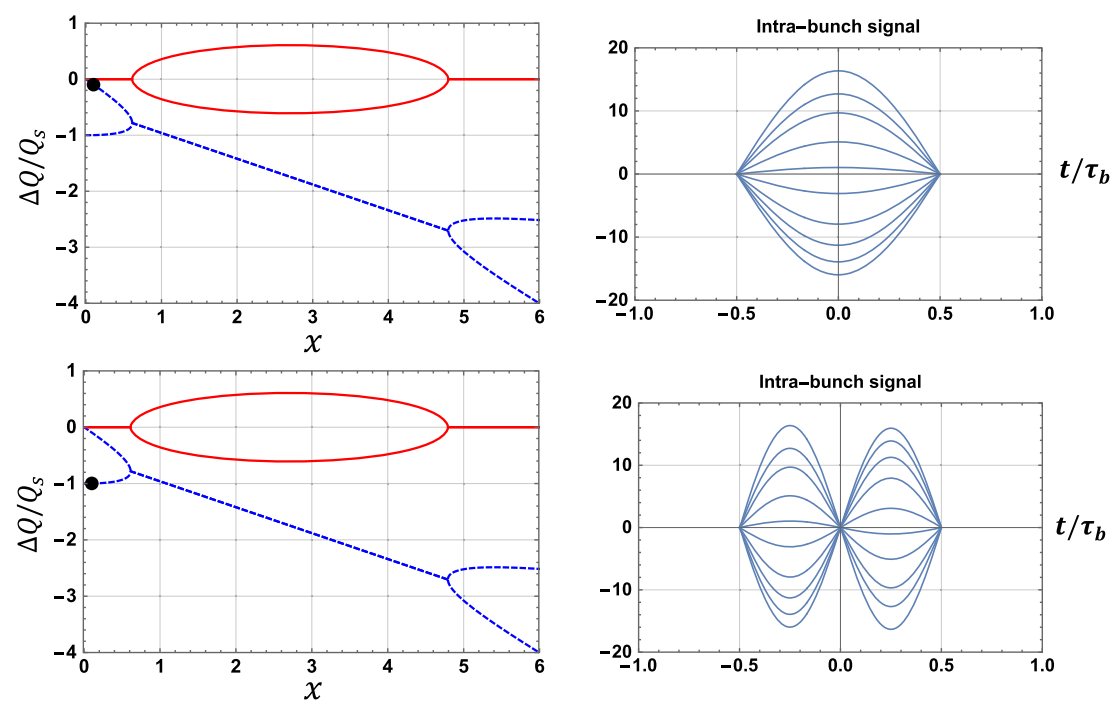

FIG. 6. Left: eigenvalues with the black dot describing the corresponding bunch intensity $(x=0.1)$, following mode 0 (top) and mode -1 (bottom); right: intrabunch signal for the mode 0 (top) and mode -1 (bottom).
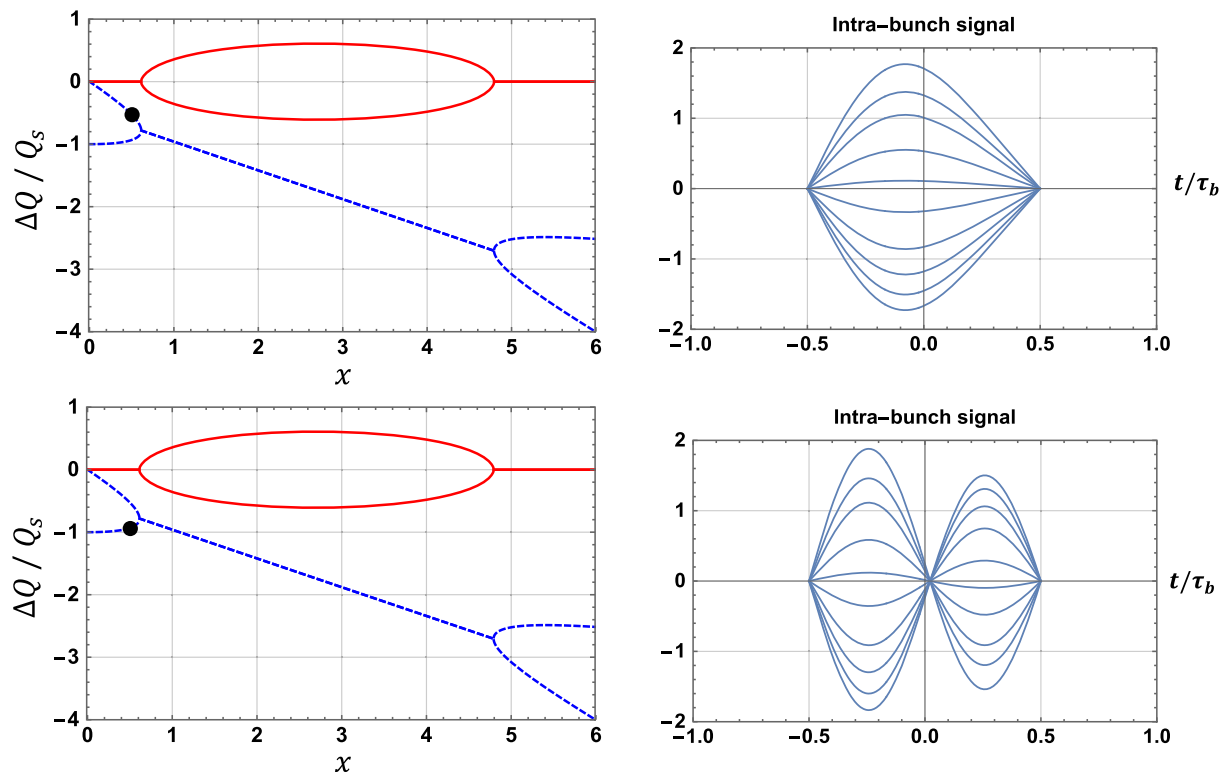

FIG. 7. Similar pictures as in Fig. 6 but for a larger bunch intensity: $x=0.5$.
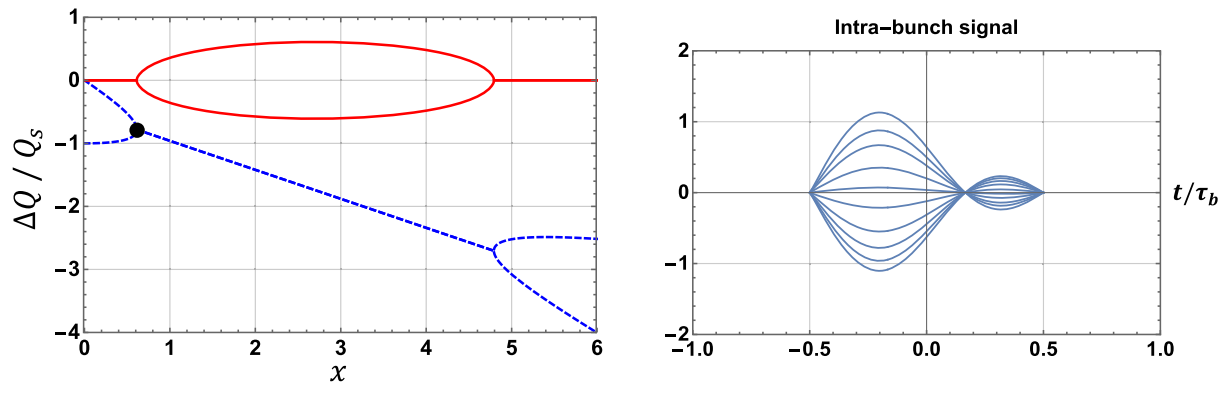

FIG. 8. Similar pictures as in Fig. 6 but for a larger bunch intensity: $x=0.61$. 
with $a_{0}=a_{-1}=a$ (see Fig. 5). The signal is zero at both bunch extremities but there is also a fixed point inside the bunch when

$$
\cos \left(\frac{\pi t}{\tau_{b}}\right)-\sin \left(\frac{2 \pi t}{\tau_{b}}\right)=0
$$

i.e., when $t=\tau_{b} / 6$ : the signal is asymmetric and shifted towards the head (see Fig. 8).

Above the TMCI intensity threshold, $S(t, n)$ is given by

$$
S(t, n) \propto(a+j b) S_{0}(t, n)-(a-j b) S_{-1}(t, n),
$$

which can be written

$$
S(t, n) \propto \sqrt{a^{2}\left[\cos \left(\frac{\pi t}{\tau_{b}}\right)-\sin \left(\frac{2 \pi t}{\tau_{b}}\right)\right]^{2}+b^{2}\left[\cos \left(\frac{\pi t}{\tau_{b}}\right)+\sin \left(\frac{2 \pi t}{\tau_{b}}\right)\right]^{2}} \cos (2 \pi n Q+\phi(t)),
$$

with $a$ and $b$ deduced from Fig. 5 ( $a$ being the imaginary part and $b$ the real one) and

$$
\phi(t)=\arctan \frac{b\left[\cos \left(\frac{\pi t}{\tau_{b}}\right)+\sin \left(\frac{2 \pi t}{\tau_{b}}\right)\right]}{a\left[\cos \left(\frac{\pi t}{\tau_{b}}\right)-\sin \left(\frac{2 \pi t}{\tau_{b}}\right)\right]} .
$$

Due to the latter term (which is coming from the fact that the eigenvectors from Fig. 5 have now both a real and an imaginary part), a traveling wave along the bunch is created: the coupling between two standing waves is a traveling wave, which is another way to see that the bunch is in the TMCI regime. The TMCI is head dominated close to the intensity threshold and the signal moves from the head to the tail when the bunch intensity is increased (see Figs. 9 and 10).
Once the modes decouple (for $x=4.8$, see Fig. 4 or Fig. 5), the signal is the symmetric of the one when the two modes couple (for $x=0.61$ ). The signal is zero at both bunch extremities but there is also a fixed point inside the bunch when

$$
\cos \left(\frac{\pi t}{\tau_{b}}\right)+\sin \left(\frac{2 \pi t}{\tau_{b}}\right)=0
$$

i.e., when $t=-\tau_{b} / 6$ : the signal is asymmetric and shifted towards the tail (see Fig. 11).

Finally, above the mode decoupling intensity threshold, the bunch is stable again (as the instability growth rate is zero, see Fig. 12), but an important amplification is revealed from head to tail, as recently discussed in the context of convective instabilities with space charge [17].
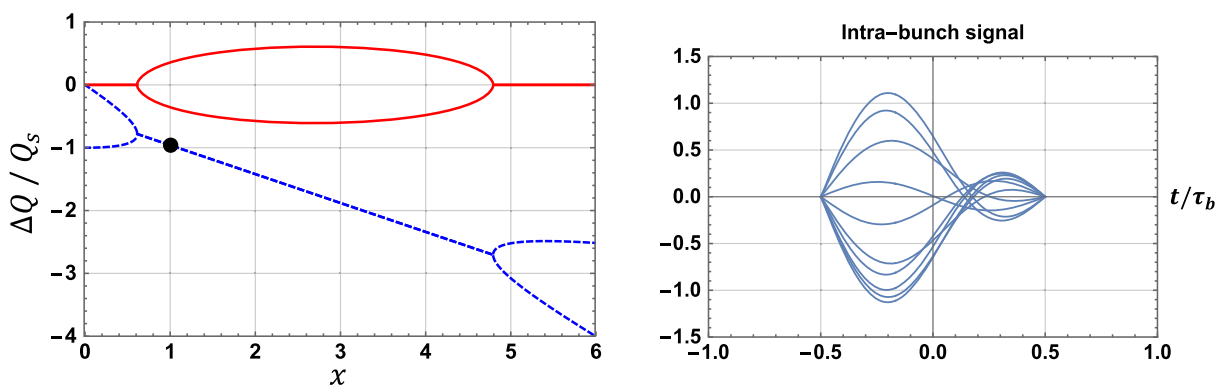

FIG. 9. Similar pictures as in Fig. 6 but for a larger bunch intensity: $x=1.0$.
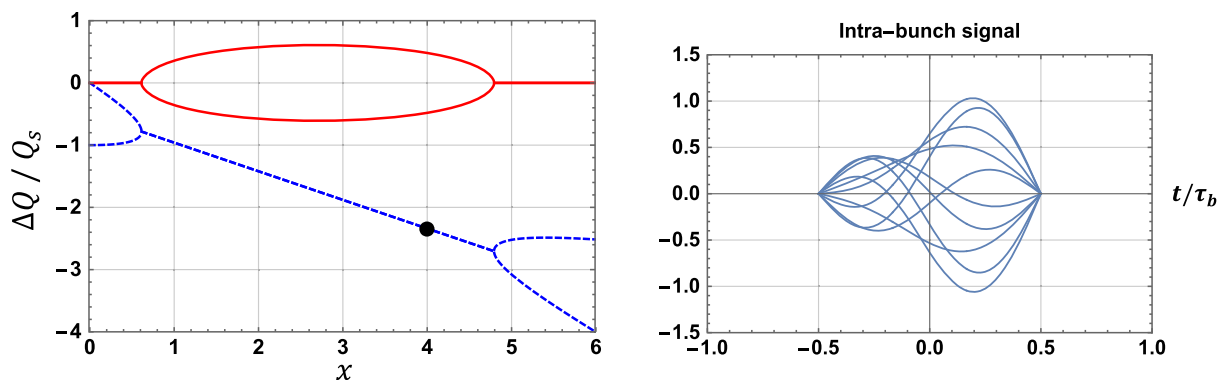

FIG. 10. Similar pictures as in Fig. 6 but for a larger bunch intensity: $x=4.0$. 

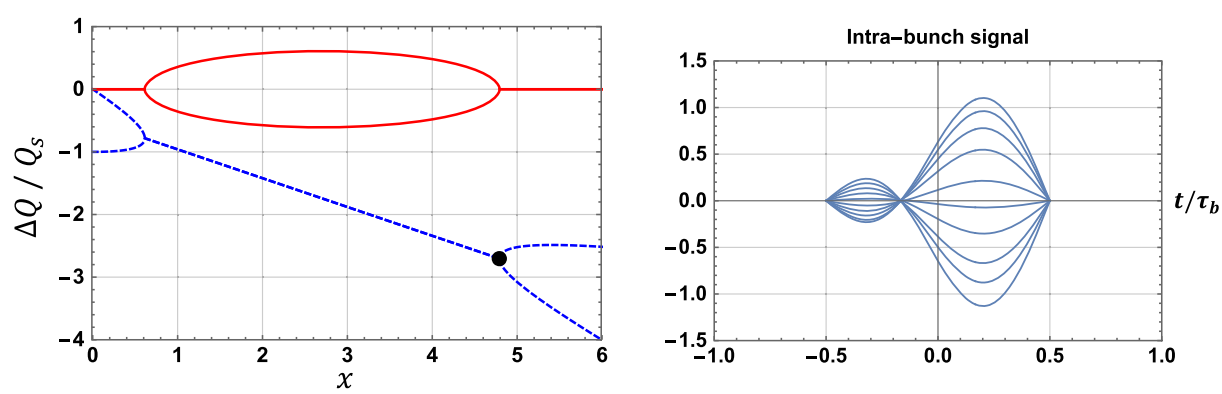

FIG. 11. Similar pictures as in Fig. 6 but for a larger bunch intensity: $x=4.8$.
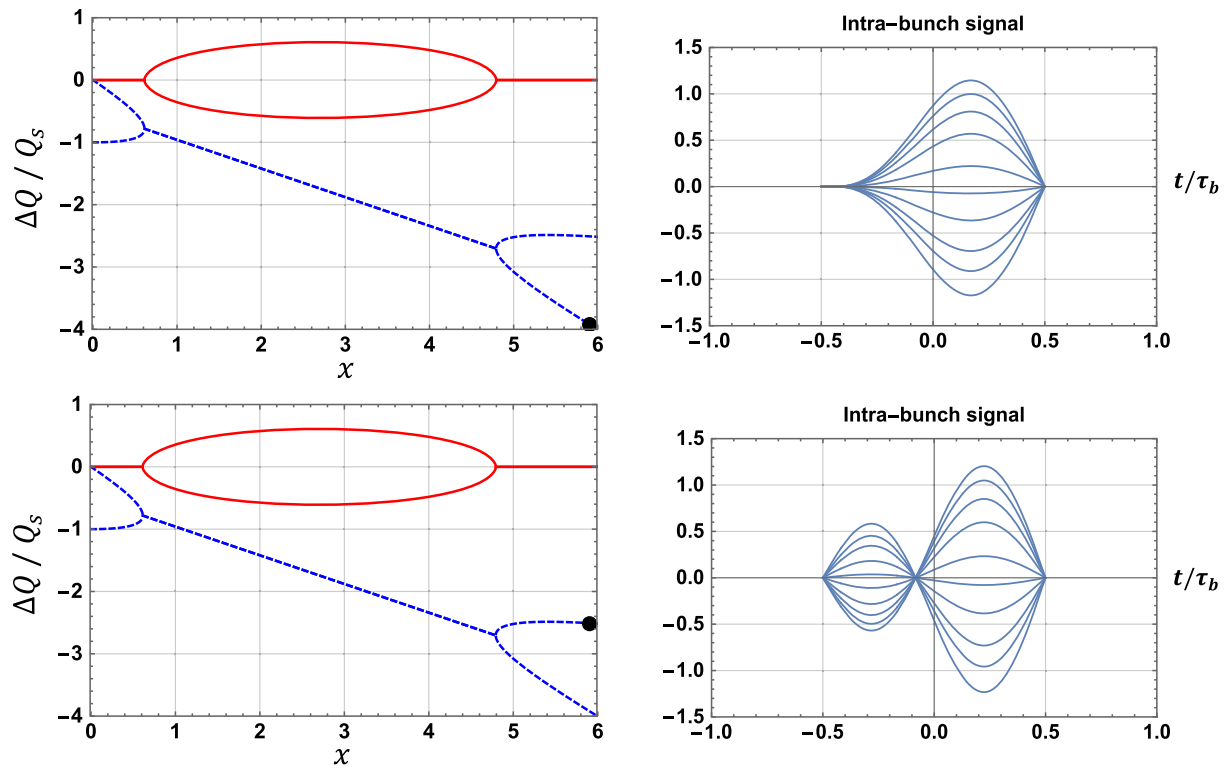

FIG. 12. Similar pictures as in Fig. 6 but for a larger bunch intensity: $x=5.9$.

\section{SIMPLE MODEL CONSIDERING TWO HIGHER-ORDER MODES TOGETHER}

A similar approach can be used to study the mode coupling between two higher-order modes, such as e.g., between modes -2 and -3 and pictures like in Fig. 13 can be obtained, revealing a huge amplification from head to tail with zero growth rate. In this case, the matrix to be diagonalized is given by

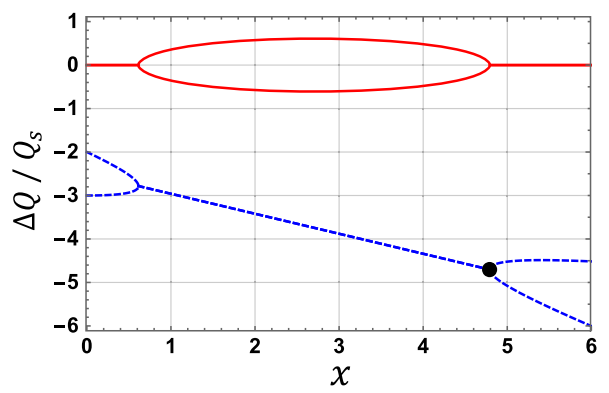

$$
H=\left[\begin{array}{cc}
-3 & -0.23 j x \\
-0.55 j x & -2-0.92 x
\end{array}\right]
$$

It is worth mentioning that the eigenvectors of Eq. (13) are the same as those of Eq. (5) (as the modes have only been shifted down by -2) and that the three fixed points of the intrabunch signal can also be computed and are solutions of

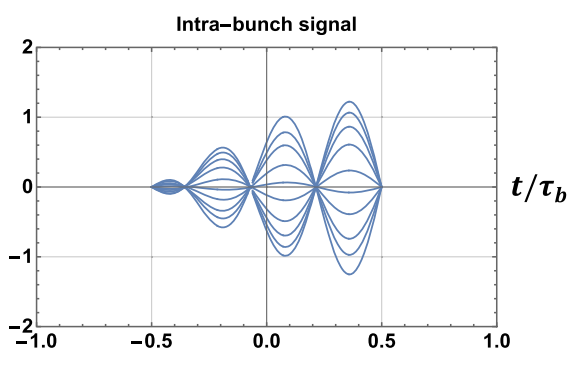

FIG. 13. Example of picture obtained in the presence of mode coupling (and mode decoupling) between modes -2 and -3 . 

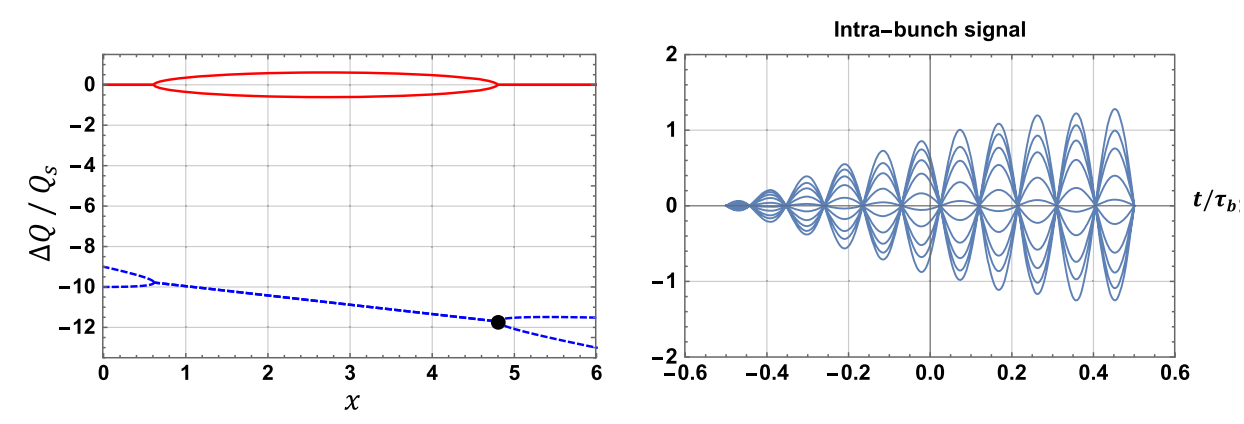

FIG. 14. Example of picture obtained in the presence of mode coupling (and mode decoupling) between modes -9 and -10 .

$$
\cos \left(\frac{3 \pi t}{\tau_{b}}\right)+\sin \left(\frac{4 \pi t}{\tau_{b}}\right)=0
$$

Another example is shown in Fig. 14 for the case of mode coupling between modes -9 and -10 , for which the matrix to be diagonalized is given by

$$
H=\left[\begin{array}{cc}
-10 & -0.23 j x \\
-0.55 j x & -9-0.92 x
\end{array}\right] \text {. }
$$

Here again the eigenvectors of Eq. (15) are the same as those of Eq. (5) (as the modes have only been shifted down by -9$)$.

\section{CONCLUSIONS}

The intrabunch motion, and its main features below, at, above the TMCI intensity threshold and also after the mode decoupling (when it exists), can be explained with a simple analytical model (considering two modes together), revealing clearly what happens when the bunch intensity is increased. The pictures of intrabunch signals obtained with this approach are very similar to the ones obtained with the DELPHI Vlasov solver [9] (compare for instance Fig. 8 obtained with the theory discussed in this paper and Fig. 3 obtained numerically from the DELPHI Vlasov solver). Furthermore, it was interesting to observe that in some cases a huge amplification factor can be observed from head

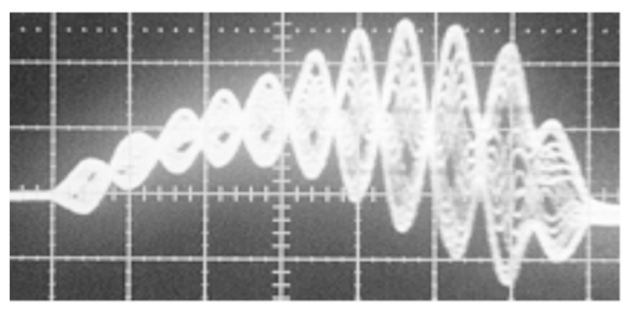

to tail with zero growth rate, as recently discussed in the context of convective instabilities with space charge [17]. A similar approach can be adopted (using the corresponding eigenvectors) with a transverse damper and/or a nonzero chromaticity and similar results are obtained [18].

This simplified model should help us to better understand the observations in the different particle accelerators and in simulations, as e.g., in Fig. 2, which clearly reveals a TMCI close to the intensity threshold as the signal is head dominated. Better characterizing an instability is the first step before trying to find the appropriate mitigation measures to push the performance of a particle accelerator. Based on the present analysis, it is proposed to use more this fundamental observable of the evolution of the intrabunch motion with intensity.

In the future, it is planned to compare the theoretical intrabunch motion (and its evolution with intensity) predicted in this paper to the one simulated with the PyHEADTAIL macroparticle tracking code for a case similar to the one discussed here, i.e., with mode coupling (between modes 0 and -1 ) and mode decoupling (see Figs. 15 and 16 of Ref. [2], where an excellent agreement was obtained between the PyHEADTAIL macroparticle tracking code and the GALACTIC Vlasov solver discussed in this paper, as concerns the mode-frequency shifts).

Furthermore, can something like this explain some past measurements in the CERN proton synchrotron (PS) and proton synchrotron booster (PSB) (in the presence of strong

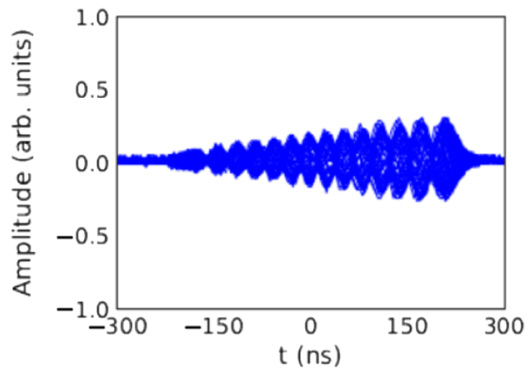

FIG. 15. Example of pictures measured in the past in the CERN PS (left) and PSB (right) in the presence of strong space charge: the head of the bunch is on the left and the tail of the bunch is on the right. This figure is courtesy of Koukovini Platia for the PSB measurements. 


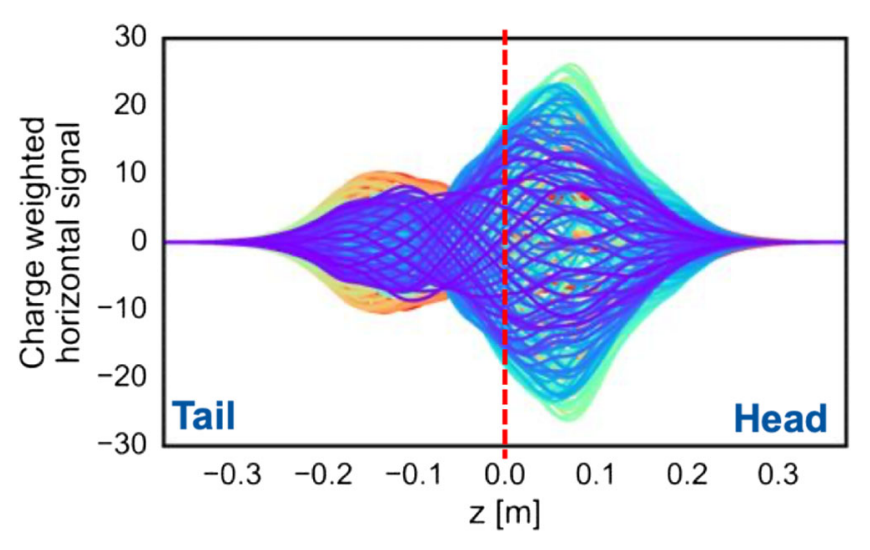

FIG. 16. Example of simulated instability in the presence of electron cloud. This figure is courtesy of Sabato.

space charge), as depicted in Fig. 15? Can something like this also explain some (parts of) simulations in the presence of electron cloud, as depicted in Fig. 16? This will be investigated in detail in the future, as these pictures look very similar to the ones produced in this paper and recent studies revealed that the effect of space charge or electron cloud can also lead to TMCI instabilities $[15,14]$, as it was already shown in the past with beam-beam [13].

[1] J. L. Laclare, Bunched beam coherent instabilities, Technical Report No. CERN-1987-003-V-1, CERN, 1987.

[2] E. Métral and M. Migliorati, Longitudinal and transverse mode coupling instability: Vlasov solvers and tracking codes, Phys. Rev. Accel. Beams 23, 071001 (2020).

[3] F. Sacherer, Single beam collective phenomena-transverse (bunched beams), Technical Report No. CERN 77-13, CERN, 1976.

[4] PyHEADTAIL, https://github.com/PyCOMPLETE/ PyHEADTAIL.

[5] M. Beck, H. Bartosik, M. Carl, K. Li, G. Rumolo, M. Schenk, and U. Van Rienen, Studies of horizontal instabilities in the CERN SPS, in 2018 International Particle Accelerator Conference (IPAC18), Vancouver, $B C$, Canada (JACoW Publishing, Geneva, Switzerland, 2018), pp. 3032-3035, https://doi.org/10.18429/JACoWIPAC2018-THPAF034.

[6] N. Mounet, Vlasov solvers and macroparticle simulations, in ICFA Mini-Workshop on Impedances and Beam Instabilities in Particle Accelerators, Benevento, Italy, edited by V. Brancolini, G. Rumolo, M. Masullo, and S. Petracca (2017), p. 77, https://doi.org/10.23732/CYRCP2018-001.77.
[7] J. Gareyte, Transverse mode coupling instabilities, AIP Conf. Proc. 592, 260 (2001).

[8] G. Rumolo, Beam instabilities, in CAS-CERN Accelerator School: Advanced Accelerator Physics, edited by W. Herr (CERN, Geneva, 2014), p. 199, https://doi.org/10.5170/ CERN-2014-009.199.

[9] D. Amorim, Study of the transverse mode coupling instability in the CERN Large Hadron Collider, Ph.D. thesis, University Grenoble Alpes, Grenoble, France, 2019, https://indico.cern.ch/event/830231/.

[10] R. D. Kohaupt, Transverse instabilities in PETRA, in 11th International Conference on High-Energy Accelerators. Experientia Supplementum, Birkhäuser, Basel, W.S. Newman (1980), Vol. 40, pp. 562-565, https://doi.org/ 10.1007/978-3-0348-5540-2_73.

[11] A. W. Chao, Physics of Collective Beam Instabilities in High Energy Accelerators (John Wiley \& Sons, New York, 1993).

[12] K. Y. Ng, Physics of Intensity Dependent Beam Instabilities (World Scientific, Singapore, 2006).

[13] S. White, X. Buffat, N. Mounet, and T. Pieloni, Transverse mode coupling instability of colliding beams, Phys. Rev. ST Accel. Beams 17, 041002 (2014).

[14] G. Iadarola, L. Mether, N. Mounet, and L. Sabato, Linearized method for the study of transverse instabilities driven by electron clouds, Phys. Rev. Accel. Beams 23, 081002 (2020).

[15] Y. Alexahin, Vlasov eigenfunction analysis of spacecharge and beam-beam effects, in ICFA Mini-Workshop on Mitigation of Coherent Beam Instabilities in Particle Accelerators, Zermatt, Switzerland, edited by E. Métral, G. Rumolo, and T. Pieloni (2020), p. 193, https://doi.org/ 10.23732/CYRCP-2020-009.

[16] T. Zolkin, A. Burov, and B. Pandey, Transverse modecoupling instability and space charge, Phys. Rev. Accel. Beams 21, 104201 (2018).

[17] A. Burov, Convective instabilities of bunched beams with space charge, Phys. Rev. Accel. Beams 22, 034202 (2019).

[18] E. Métral, Intra-bunch motion, Technical Report No. CERN-ACC-NOTE-2020-0018, CERN, 2020.

[19] E. Métral, D. Amorim, S. Antipov, N. Biancacci, X. Buffat, and K. Li, Destabilizing effect of the LHC transverse damper, in 9th International Particle Accelerator Conference (IPAC18), Vancouver, Canada (JACoW Publishing, Geneva, Switzerland, 2018), p. 3076, https:// doi.org/10.18429/JACoW-IPAC2018-THPAF048.

[20] E. Métral, Destabilizing effect of resistive transverse dampers, in ICFA Mini-Workshop on Mitigation of Coherent Beam Instabilities in Particle Accelerators, Zermatt, Switzerland, edited by E. Métral, G. Rumolo, and T. Pieloni (2020), p. 221, https://doi.org/10.23732/ CYRCP-2020-009. 